\title{
Changes in flavor compounds of Aloididae aloidi during enzymatic hydrolysis
}

\author{
Xuting Bai ${ }^{1}$, Tao $\mathrm{Li}^{1}$, Honglei Zhao ${ }^{1 *}$, Xuepeng $\mathrm{Li}^{1}$, Wenhui $\mathrm{Zhu}^{1}$, Ying $\mathrm{Bu}^{1}$, Jianrong $\mathrm{Li}^{1}$, Yongxia $\mathrm{Xu}^{1 *}$ \\ ${ }^{1}$ College of Food Science and Technology, Laboratory Center, Bohai University, National \& Local Joint Engineering Research Center of \\ Storage, Processing and Safety Control Technology for Fresh Agricultural and Aquatic Products, Jinzhou 121013, China
}

\begin{abstract}
Protamex was selected to prepare the hydrolysate. E-tongue, free amino acid combined with soluble peptide analysis were used to detect the flavor changes of Aloididae aloidi during enzymolysis. Degree of proteolysis increased with the prolongation of enzymolysis time, and reached the maximum value at 8 hours. The content of soluble peptide of hydrolysate increased firstly and then decreased in the later process. The E-tongue could effectively distinguish the taste difference of hydrolysates at different enzymolysis time, and the hydrolysate presented strong bitterness and astringency during the whole enzymolysis. The total amount of free amino acids in the hydrolysate increased gradually, and some sweet, umami and bitter amino acids increased in varying degrees during the process of enzymolysis.
\end{abstract}

\section{Introduction}

The blue clam (Aloididae aloidi), a small low-value shellfish, is abundant in the coastal areas of China. However, due to its small size and difficulty in processing, most of them are processed into aquaculture bait except for a small amount for fresh food, resulting in a great waste of resources [1]. The protein content of blue clam is up to $60 \%$, and the amino acid composition is very comprehensive. The total amount of amino acids and essential amino acids in blue clam are higher than those in oyster and equivalent to mussels. In addition, taste amino acids account for more than $50 \%$ of the total amino acids in blue clam protein, among which alanine and glycine are higher, which are the high-quality raw materials for seafood flavoring.

Biological enzymolysis technology is a common method to produce natural and safe seasoning base materials. It can not only fully hydrolyze the raw protein, but also has many advantages such as mild reaction conditions, low energy consumption, less pollution, directional and controllable reaction process. It is an important mean of high-value utilization of valuable fish and shrimp. In the process of enzymatic hydrolysis, the tertiary structure of proteins is gradually dissociated and their conformation is destroyed. A great many of flavoring substances such as free amino acids and short chain peptides are produced, which are more and more favored by people. At present, the commercial proteases used for enzymatic hydrolysis of aquatic proteins mainly include neutral, alkaline, compound, papaya and flavor proteases. Due to the substrate specificity of proteases, different proteases act on the peptide chain at different sites, which also have an important impact on the flavor composition and nutritional characteristics of the enzymatic hydrolysate [2]. In view of this, this paper selected complex protease enzyme hydrolysis of blue clam protein, using electronic tongue, TCA-soluble peptide and free amino acids and other indicators to analyze the changes of flavor substances of blue clam during enzymatic hydrolysis.

\section{Materials and methods}

\subsection{Materials}

Fresh blue clams were obtained from aquatic products market in Linxi Street (Jinzhou City, Liaoning, China). The blue clams are quick-frozen after spitting in sand, and the shells were removed in the frozen state to take the meat and set aside.

Protease is of food grade and purchased from Nanning Pangbo Enzyme Preparation Co., Ltd. (Guangxi, China). o-phthalaldehyde and methanol are all analytically pure and purchased from Sinopharm Reagent Co., Ltd. (Shanghai, China).

\subsection{Preparation of blue clam enzymatic hydrolysate}

Blue clam muscles $(30 \mathrm{~g})$ was added with $90 \mathrm{~mL}$ distilled water and thoroughly mixed. Then the enzyme was deactivated at $90^{\circ} \mathrm{C}$ water bath for $15 \mathrm{~min}$. After cooling to $40^{\circ} \mathrm{C}$, the optimal $\mathrm{pH}$ range of protease was adjusted. Then $0.3 \%$ compound protease was added, and the mixture was fully mixed and hydrolysate $8 \mathrm{~h}$ at the optimum temperature. After enzymolysis, the enzyme was deactivated and cooled to room temperature. The

\footnotetext{
Corresponding author: cristy320@163.com
} 
enzymatic hydrolysate was centrifuged at $5000 \times \mathrm{g}$ for $10 \mathrm{~min}$, and the supernatant was taken for standby.

\subsection{Determination of hydrolysis degree}

The degree of hydrolysis of the enzymatic hydrolysate was determined by the o-phthalaldehyde method [3].

\subsection{Determination of soluble peptide content}

The enzymatic hydrolysate $(5 \mathrm{~mL})$ was fully mixed with $15 \mathrm{~mL} 5 \%(\mathrm{~W} / \mathrm{V})$ trichloroacetic acid (TCA) solution, and the supernatant was centrifuged for 30 min. $4 \mathrm{~mL}$ biuret reagent was added to $1 \mathrm{~mL}$ supernatant, shaken well, stood at $25^{\circ} \mathrm{C}$ for $30 \mathrm{~min}$, and the absorbance at $540 \mathrm{~nm}$ was determined.

\subsection{Electronic tongue detection}

The enzymatic hydrolysate samples $(35 \mathrm{~mL})$ were placed in the electronic tongue special sample cup, and put them on the electronic tongue automatic sampler for detection according to the set sequence. The single sampling time is $120 \mathrm{~s}, 1$ time/s. Each group of samples was tested repeatedly 4 times, and after 3 times the sensor signal became stable, that is, the response value of each sensor at the 120th s was analyzed for taste characteristics.

\subsection{Determination of free amino acids}

Amino acid analysis was done by using an automatic amino acid analyzer (L-8900; Hitachi, Tokyo, Japan). 1 $\mathrm{mL}$ enzymatic hydrolysate was centrifugated in a high-speed refrigerated centrifuge $(12000 \times \mathrm{g}, 10 \mathrm{~min}$; Avanti J-26XP, Beckman, Shanghai, China). The supernatant was filtered through a $20 \mu \mathrm{m}$ filter membrane and then injected into an automatic amino acid analyzer.

\section{Results and discussion}

\subsection{Changes in the degree of hydrolysis}

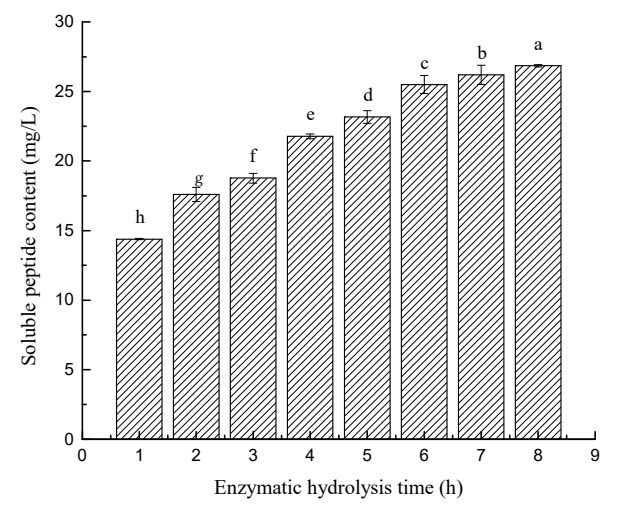

Fig. 1 Changes of hydrolysis degree of Aloididae aloidi during enzymolysis
The changes of hydrolysis degree during enzymatic hydrolysis of blue clam are shown in Fig. 1. With the increase of enzymolysis time, the degree of hydrolysis increased gradually, and reached the maximum value of $26.9 \%$ at $8 \mathrm{~h}$, indicating that the blue clam obtained better enzymatic hydrolysis. Compound protease is a high-efficiency hydrolase, which is widely used in the preparation of aquatic products and animal protein hydrolysates [4]. Yao et al. [5] found that the hydrolysis degree of low value freshwater fish can reach a higher degree of hydrolysis after optimization. During the enzymolysis process, the protease acted on the cleavage site of the blue clam, and the stability of the subunits was destroyed. With the increase of enzymolysis time, the sites of enzyme action on the protein molecules decreased gradually with the extension of time and the accumulation of enzymatic hydrolysis products also inhibited the progress of enzymatic reaction.

\subsection{Changes in soluble peptide content}

TCA soluble peptides can well reflect the content of short peptide chains in foods, and can be used as one of the indicators to evaluate food nutrition. Fig. 2 shows that the content of TCA soluble peptides increased first and then decreased with time during the enzymolysis process. During the process of enzymatic hydrolysis, with the increase of enzymatic hydrolysis time, the secondary and tertiary structures of protein were destroyed under the action of complex protease, the tissues were dissociated, and the soluble peptide content increased. As the further enzymatic hydrolysis progressed, the soluble peptide was hydrolyzed into free amino acids, thus the content of which tended to decrease.

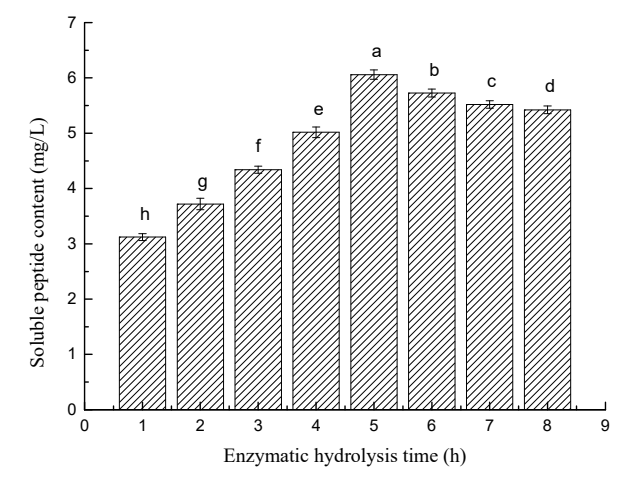

Fig. 2 Changes of soluble peptide of Aloididae aloidi during enzymolysis

\subsection{Electronic tongue analysis}

Electronic tongue is a new method for analyzing and detecting the taste of liquid developed rapidly in recent years. It can simulate the human tongue to evaluate the acidity, bitterness, saltiness, freshness, astringency, aftertaste and richness of samples [6]. The electronic tongue is used to describe the overall flavor profile during enzymatic hydrolysis of blue clams. The response values of the electronic tongue are shown in Fig. 3. It 
can be seen that the response values of bitterness, astringency, and saltiness were more prominent in enzymolysis solution, indicating that the bitterness and astringency substances play an important role in the taste of enzymolysis solution. During enzymatic hydrolysis, the protein conformation was destroyed under the action of enzymes, and shorter peptide chains were gradually formed. Certain peptides with hydrophobic groups on the side chains could cause severe bitterness [7]. In addition, blue clams produced a large amount of arginine during enzymatic hydrolysis, which has a certain bitter taste. Therefore, the bitter taste of the enzymatic hydrolysate might also be derived from the formation of a large amount of bitter amino acids. Fig. 4 is the PCA of the electronic tongue response value of blue clam enzymatic hydrolysate, where the first principal component is $69.5 \%$ and the second principal component is $18.5 \%$. The sum of the first and second principal components exceeded $85 \%$, indicating that it could represent most of the information about the changes in flavor substances of blue clams during enzymatic hydrolysis, and could reflect the law of flavor changes.

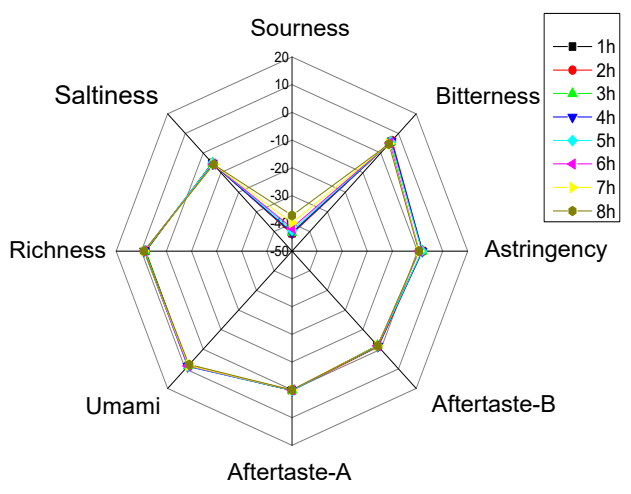

Fig. 3 Radar diagram of electronic tongue of Aloididae aloidi during enzymolysis

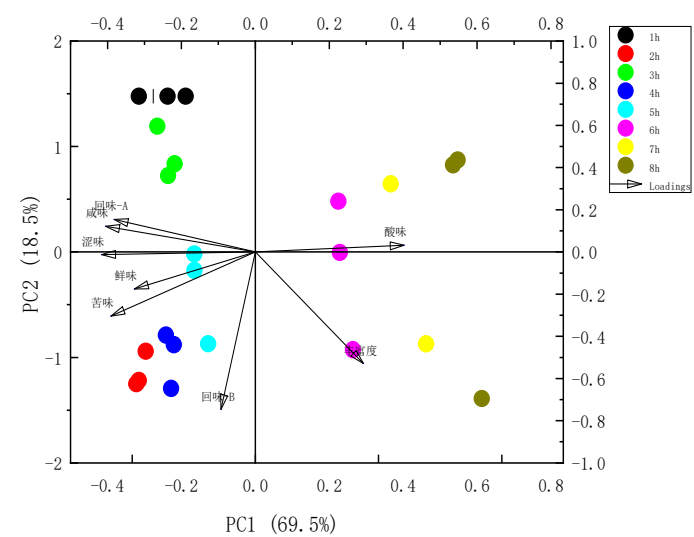

Fig. 4 Principal component analysis of electronic tongue of Aloididae aloidi during enzymolysis

\subsection{Changes of free amino acid content}

The composition and content of amino acids play a very important influence on the taste characteristics of the enzymatic hydrolysate [8]. The changes of free amino acids in blue clam during enzymolysis are shown in Table 1. It can be seen that the content of free amino acids in the enzymatic hydrolysate of blue clam gradually increased with time. The total content of free amino acids reached the maximum at $7 \mathrm{~h}$ of hydrolysis, and showed a downward trend at $8 \mathrm{~h}$, which might be due to the fact that the hydrolysis degree of the enzymatic hydrolysate was close to saturation at $7 \mathrm{~h}$ of hydrolysis. Fifteen amino acids were detected in blue clam hydrolysate, including two umami amino acids, four sweet amino acids and eight bitter amino acids. With the increase of enzymatic hydrolysis time, sweet, bitter and umami amino acids increased in different degrees. Both aspartic acid and glutamic acid exhibit umami flavor and are the important raw materials for the preparation of umami flavor agents [9]. The four sweet amino acids of glycine, serine, alanine and threonine also have a certain promotion effect on the flavor of the enzyme hydrolysate. Among them, glycine not only has sweet taste, but also can reduce the bitter taste in food and promote the taste sensation of blue clam enzymatic hydrolysate. The total amount of bitter amino acids was always relatively high during the enzymatic hydrolysis, and the content of arginine was the highest, reaching $256.3 \mathrm{mg} / 100 \mathrm{~g}$ during the enzymatic hydrolysis, which was consistent with the result as previously reported [10]. Arginine is a bitter amino acid, which could make a strong bitter taste of the enzymatic hydrolysate affect its sensory quality. Meanwhile, the synergistic effect of various bitter amino acids in the enzymatic hydrolysate could also intensify the bitter taste of the product.

Table 1 Changes of free amino acids in Aloididae aloidi during enzymolysis

\begin{tabular}{|c|c|c|c|c|c|c|c|c|}
\hline \multirow{2}{*}{ Free amino acids } & \multicolumn{8}{|c|}{ Concentration $(\mathrm{mg} / 100 \mathrm{~g})$} \\
\hline & $1 \mathrm{~h}$ & $2 \mathrm{~h}$ & $3 \mathrm{~h}$ & $4 \mathrm{~h}$ & $5 \mathrm{~h}$ & $6 \mathrm{~h}$ & $7 \mathrm{~h}$ & $8 \mathrm{~h}$ \\
\hline Phe & 76 & 82 & 96 & 76 & 124 & 138 & 138 & 130 \\
\hline Ala & 42 & 44 & 44 & 40 & 50 & 50 & 52 & 52 \\
\hline Met & 19 & 22 & 26 & 24 & 36 & 40 & 44 & 42 \\
\hline Gly & 16 & 16 & 16 & 14 & 16 & 16 & 16 & 16 \\
\hline Glu & 28 & 24 & 24 & 28 & 32 & 38 & 34 & 36 \\
\hline Arg & 146 & 162 & 192 & 166 & 240 & 256 & 256 & 252 \\
\hline Lys & 88 & 96 & 116 & 108 & 158 & 168 & 176 & 174 \\
\hline Tyr & 69 & 66 & 74 & 68 & 94 & 116 & 110 & 102 \\
\hline Leu & 70 & 82 & 98 & 88 & 140 & 154 & 158 & 158 \\
\hline Ser & 13 & 9 & 10 & 13 & 17 & 20 & 22 & 24 \\
\hline Thr & 42 & 42 & 48 & 48 & 70 & 80 & 84 & 84 \\
\hline Asp & 3 & 4 & 4 & 5 & 7 & 8 & 8 & 9 \\
\hline Val & 26 & 30 & 36 & 34 & 52 & 59 & 64 & 60 \\
\hline Ile & 30 & 33 & 39 & 38 & 56 & 64 & 68 & 66 \\
\hline His & 10 & 17 & 18 & 19 & 24 & 28 & 30 & 24 \\
\hline
\end{tabular}

\section{Conclusions}

The changes of flavor components of blue clams enzymatic hydrolysate by protamex were studied. The 
results showed that the hydrolysis degree gradually increased with the extension of enzymatic hydrolysis time, which reached the maximum value at $8 \mathrm{~h}$ of enzymatic hydrolysis. The soluble peptide content increased first and then decreased during enzymolysis. The electronic tongue could obviously distinguish the taste difference of the enzymatic hydrolysate under different enzymolysis time, and the enzymatic hydrolysate exibited a strong bitterness and astringency. With the extension of the enzymolysis time, the total amount of free amino acids in enzymatic hydrolysate gradually increased, and some sweet, umami and bitter amino acids increased in different degrees during the process of enzymolysis.

\section{Acknowledgements}

This work was supported by the National Key R\&D Program of China (2016YFD0400705), the fund of the Beijing Key Laboratory of Flavor Chemistry, Beijing Technology and Business University (SPFW2020YB07), and the First-class Discipline Project of Liaoning Province (LNSPXKBD2020315).

\section{References}

1. Y. Bu, L. W. Zhu, W. T. Xu, W. H. Zhu, H. Liu, J. R. Li, X. P. Li. Int. J. Food Sci. Tech. DOI: 10.1111/ijfs.14802 (2020)

2. A. W. Mohammad, A. G. Kumar, R. K. Basha. Int Aquat Res 7, 27-39 (2015)

3. C. F. Chi, B. Wang, F. Y. Hu, Y. M. Wang, B. Zhang, S. G. Deng, W. Wu. Food Res. Int 73, 124-129 (2015)

4. L. Feng, Y. Qiao, Y. F. Zou, M. Huang, Z. L. Kang, G. H. Zhou. Meat Sci 98, 34-40 (2014)

5. N. Bhaskar, T. Benila, C. Radha, R. G. Lalitha. Bioresource Technol 99, 335-343 (2006)

6. K. Toko. Biosens Bioelectron 13, 710-709 (1998)

7. H. O. Kim, E. C. Li-Chan. J. Agric. Food Chem 54, 10102-10111 (2006)

8. G. L. Mo, J. W. Chen, J. B. Liu, J. Shu, E. Liao, W. S. Xia, S. Y. Cheng. Food Sci 40, 23-28 (2019)

9. S. Yamaguchi, T. Yoshikawa, S. Ikeda, T. Ninomiya. J. Food Sci 36, 846-849 (2010)

10. H. N. Lioe, A. Apriyantono, K. T. Kojiwada, M. Yasuda. J. Food Sci 70, 401-405 (2005) 